Buul, L.W. van, Veenhuizen, R.B., Achterberg, W.P., Schellevis, F.G., Essink, R.T.G.M., Greeff, S.C. de, Natsch, S., Steen, J.T. van der, Hertogh, C.M.P.M. Antibiotic prescribing in Dutch nursing homes: how appropriate is it? JAMDA: Journal of the American Medical Directors Association: 2015, 16(3), 229-237

\begin{tabular}{l|l}
$\begin{array}{l}\text { Postprint } \\
\text { Version }\end{array}$ & 1.0 \\
\hline Journal website & http://www.jamda.com/article/S1525-8610(14)00621-5/abstract \\
\hline Pubmed link & http://www.ncbi.nlm.nih.gov/pubmed/25458444 \\
\hline DOI & $10.1016 /$ j.jamda.2014.10.003
\end{tabular}

This is a NIVEL certified Post Print, more info at http://www.nivel.eu

\title{
Antibiotic Prescribing In Dutch Nursing Homes: How Appropriate Is It?
}

Laura W. Van BuUl, MSC ${ }^{A, B}$, Ruth B. Veenhuizen, MD, PhD ${ }^{A, B}$, Wilco P. ACHTERBERG, MD, PHD ${ }^{\mathrm{C}}$, FrANÇOIS G. SCHELLEVIS, MD, PHD ${ }^{\mathrm{A}, \mathrm{B}, \mathrm{D}}$, ROB T.G.M. EsSinK, MPH, PharmD ${ }^{\mathrm{E}}$, SABine C. DE GreEfF, PhD ${ }^{\mathrm{F}}$, StePhanie NATSCh, PharmD, PhD ${ }^{\mathrm{G}}$, Jenny T. VAN DER STEEN, PHD ${ }^{A, B,}$, CeEs M.P.M. HeRTOGH, MD, PHD ${ }^{A, B}$

${ }^{a}$ EMGO Institute for Health and Care Research, VU University Medical Center, Amsterdam, The Netherlands

${ }^{\mathrm{b}}$ Department of General Practice and Elderly Care Medicine, VU University Medical Center, Amsterdam, The Netherlands

${ }^{c}$ Department of Public Health and Primary Care, Leiden University Medical Center, Leiden, The Netherlands

${ }^{d}$ NIVEL (Netherlands Institute for Health Services Research), Utrecht, The Netherlands

${ }^{\mathrm{e}}$ Dutch Institute for Rational Use of Medicine (IVM), Utrecht, The Netherlands

${ }^{f}$ Centre for Infectious Disease Control (Clb), National Institute for Public Health and the Environment (RIVM), Bilthoven, The Netherlands

${ }^{g}$ Department of Pharmacy, Radboud University Medical Center, Nijmegen, The Netherlands

\begin{abstract}
Objective: To investigate the appropriateness of decisions to prescribe or withhold antibiotics for nursing home $(\mathrm{NH})$ residents with infections of the urinary tract (UTI), respiratory tract (RTI), and skin (SI). Design: Prospective study. Setting: Ten NHs in the central-west region of the Netherlands.

Participants: Physicians providing medical care to NH residents. Measurements: Physicians completed a registration form for any suspected infection over an 8month period, including patient characteristics, signs and symptoms, and treatment decisions. An algorithm, developed by an expert panel and based on national and international guidelines, was used to evaluate treatment decisions for appropriateness of initiating or withholding antibiotics. Results: Appropriateness of 598 treatment decisions was assessed. Overall, 76\% were appropriate, with cases that were prescribed antibiotics judged less frequently "appropriate" (74\%) compared with cases in which antibiotics were withheld (90\%) $(P=.003)$. Decisions around UTI were least often appropriate (68\%, compared with $87 \%$ for RTI and $94 \%$ for SI $[P<.001])$. The most common situations in which antibiotic prescribing was considered inappropriate were those indicative of asymptomatic bacteriuria or viral RTI.
\end{abstract}


Buul, L.W. van, Veenhuizen, R.B., Achterberg, W.P., Schellevis, F.G., Essink, R.T.G.M., Greeff, S.C. de, Natsch, S., Steen, J.T. van der, Hertogh, C.M.P.M. Antibiotic prescribing in Dutch nursing homes: how appropriate is it? JAMDA: Journal of the American Medical Director Association: 2015, 16(3), 229-237

Conclusion: Although the rate of appropriate antibiotic prescribing in Dutch NHs is relatively high compared with previous studies in other countries, our results suggest that antibiotic consumption can be reduced by improving appropriateness of treatment decisions, especially for UTI. Given the current antibiotic resistance developments in long-term care facilities, interventions reducing antibiotic use for asymptomatic bacteriuria and viral RTI are warranted.

Antibiotics are one of the most commonly prescribed drug classes in long-term care facilities (LTCFs), with $47 \%$ to $79 \%$ of the residents receiving at least 1 course of antibiotics annually. The substantial antibiotic use contributes to the development of antibiotic resistance in this setting. ${ }^{1}$ and 2 In addition, there is increasing evidence that LTCFs serve as a reservoir for transmission of resistant organisms to other health care settings. ${ }^{3,4}$ and 5 Infections with antibiotic-resistant organisms in LTCFs have been associated with increased morbidity, mortality, and costs. This has raised awareness of the importance of strategies to reduce antibiotic resistance, including the promotion of appropriate use of antibiotics. ${ }^{2}$

To increase appropriate antibiotic prescribing, we need insight into the degree and nature of inappropriate use. Previous studies in LTCFs reported that, overall, decisions to start antibiotic treatment were appropriate in $49 \%$ to $63 \%$ of cases. ${ }^{6,7,8,9 \text {, }}$ 10 and 11 For specific infections, 2 American studies reported that criteria to start antibiotic treatment were met in $19 \%$ and $27 \%$ of urinary tract infections (UTIs), ${ }^{12}$ and 13 and 1 study found that initiation of antibiotics was justified in $81 \%$ of respiratory tract infections (RTIs). ${ }^{14}$ Some studies used guideline-based criteria to judge appropriateness of antibiotic prescribing. ${ }^{6,7,11 \text { and } 14}$ Others used the criteria developed by McGeer et $\mathrm{al}^{15}$ to assess appropriateness. ${ }^{8,9,10,12 \text { and } 13}$ Although the latter are widely recognized criteria, they have been developed for infection surveillance purposes and are therefore highly specific rather than highly sensitive. Some argue that these criteria should therefore not be used to assess the appropriateness of initiating antibiotic treatment. ${ }^{5}$ Further, previous studies relied on patient chart review to assess clinical features, whereas charts may not always reliably reflect the actual clinical situation. For example, Zimmer et $\mathrm{al}^{6}$ reported that signs and symptoms were registered in patient charts in fewer than half of the cases.

We investigated the appropriateness of decisions to prescribe or withhold antibiotics for nursing home $(\mathrm{NH})$ residents, based on registration forms completed by physicians at the time of diagnosing an infection. The study was conducted in NHs in the Netherlands, where antibiotic consumption in primary care is low compared with other European Union countries, ${ }^{16}$ but where antibiotic consumption in NHs is comparable to European means. ${ }^{17}$ We quantified appropriateness of decisions to prescribe or withhold antibiotics in Dutch NHs, and investigated if this varied among physicians and if this was associated with patients' characteristics. Further, we identified common clinical situations in which antibiotics are prescribed inappropriately. 
Buul, L.W. van, Veenhuizen, R.B., Achterberg, W.P., Schellevis, F.G., Essink, R.T.G.M., Greeff, S.C. de, Natsch, S., Steen, J.T. van der, Hertogh, C.M.P.M. Antibiotic prescribing in Dutch nursing homes: how appropriate is it? JAMDA: Journal of the American Medical Director Association: 2015, 16(3), 229-237

\section{METHODS}

\section{Study Setting}

The study was conducted in 10 NHs participating in a research project aimed at rationalizing antibiotic prescribing in LTCFs: the Improving Rational Prescribing of Antibiotics in Long-term Care Facilities (IMPACT) study. ${ }^{18}$ The current study comprises a baseline measurement, ahead of any intervening to improve antibiotic prescribing. Table 1 summarizes the recruitment of study facilities. Eight NHs were located in urban areas, and 2 were located in rural areas, all in the central-west region of the Netherlands. In the Netherlands, NHs employ elderly care physicians (formerly called nursing home physicians), which is a distinct medical specialty in the Netherlands. These physicians have the NH as their main, and often only, site of practice. Dutch NHs accommodate residents on 3 types of wards: somatic wards, for physically disabled residents; psychogeriatric wards, predominantly for residents suffering from dementia; and rehabilitation wards. ${ }^{19}$ Regarding infection management, hospitalization and the administration of intravenous fluids or drugs are rare in Dutch NHs. ${ }^{20}$

\section{[TABLE 1]}

\section{Data Collection}

Physicians providing medical care to residents completed a registration form in case they, based on their clinical judgment, suspected a UTI, an RTI, or a skin infection (SI). Infections were registered over an 8-month period, as soon as possible after the consultation, and regardless of whether antibiotic treatment was initiated. In 9 NHs, this 8-month period occurred between January 2012 and October 2012. In 1 NH, due to organizational issues, data collection was delayed and occurred between April 2012 and December 2012. The registration form included documentation of the following: patient characteristics (eg, age, sex, wheelchair dependence), vital signs in the past 48 hours (eg, blood pressure, pulse, temperature), recent/current health status (eg, new or worsening confusion, decreased intake), medical history (eg, diabetes, chronic obstructive pulmonary disease [COPD], dementia), signs and symptoms related to the suspected infection type, and details of the treatment decision (ie, antibiotic prescribing, including details on the prescription, or no antibiotic prescribing including the reason for not prescribing). Recurrent infections were included, as cases were defined as infection consultations rather than patients. Only infections diagnosed in the $\mathrm{NH}$ were included. In case an infection was diagnosed by an on-call physician not employed by the $\mathrm{NH}$, the employed physician responsible for the care of the patient completed the registration form based on the descriptions of the on-call physician.

Chart review was conducted to identify missing cases (ie, infection consultations for which physicians did not complete a registration form), and to investigate whether these cases were comparable to those registered. To this end, we selected a random sample of residents and invited these residents or, if not mentally competent, a family member to provide written consent to review their charts. On average, 32\% (range $22 \%-49 \%$ ) of the residents were selected. Two researchers (LB and SD) screened 
Buul, L.W. van, Veenhuizen, R.B., Achterberg, W.P., Schellevis, F.G., Essink, R.T.G.M., Greeff, S.C. de, Natsch, S., Steen, J.T. van der, Hertogh, C.M.P.M. Antibiotic prescribing in Dutch nursing homes: how appropriate is it? JAMDA: Journal of the American Medical Director Association: 2015, 16(3), 229-237

patient charts of consenting residents for infection consultations, over the same 8month period during which physicians completed registration forms.

\section{Appropriateness of Treatment Decisions}

We developed an algorithm for each infection type to evaluate appropriateness of initiating or withholding antibiotics (Appendix). These algorithms were based on consensus within the research team and a national expert panel, and they were founded on national evidence-based guidelines (of the Dutch College of General Practitioners, and the Dutch Association of Elderly Care Physicians and Social Geriatricians) and an international consensus-driven guideline. ${ }^{21}$ The national expert panel comprised 2 infectious disease specialists, 4 general practitioners with extensive expertise in infectious diseases, an infectious diseases researcher, an infectious diseases epidemiologist, an elderly care physician with extensive expertise in infectious diseases, and 2 medical microbiologists. The algorithm classified treatment decisions as (1) appropriate, (2) probably appropriate, (3) probably inappropriate, (4) inappropriate, or (5) insufficient information to evaluate the treatment decision. A treatment decision was judged "(in)appropriate” if there was strong evidence for this judgment, and "probably (in)appropriate" if the evidence was less strong but still sufficient for this judgment. Two researchers (LB and RV) assessed the first 181 physician-registered infections together, to achieve consistency of evaluations. The remaining cases were assessed by each researcher independently. In case of doubt or disagreement, the researchers discussed their judgments to achieve consensus, in some cases in a project team meeting.

\section{Data Analysis}

The data on the infection registration forms were entered into a Microsoft Access 2000 (Microsoft Corporation, Redmond, WA) database by 2 persons independently. The data were subsequently processed in SPSS version 20 (IBM Corporation, New York, NY). We used descriptive statistics to summarize the data. The dichotomous variable "appropriateness" was created based on the conclusions of the algorithm, by combining "appropriate" and "probably appropriate" into "appropriate," and “inappropriate” and "probably inappropriate” into "inappropriate.” Chi-square tests, analysis of variance, and Kruskal-Wallis tests were used as appropriate, to analyze differences between facilities in demographic characteristics and appropriateness of treatment decisions, differences between infection types in appropriateness of treatment decisions, and differences between infections treated and not treated with antibiotics in appropriateness of treatment decisions. To investigate our hypothesis that appropriate prescribing may vary among physicians and may be associated with type of unit, dementia, urinary catheter (for UTI), and COPD (for RTI), a secondorder penalized quasilikelihood multilevel logistic regression analysis was performed using MLwiN version 2.30 (Centre for Multilevel Modelling, University of Bristol, Bristol, UK). In this model, the data were clustered in 3 levels: NH, patient, and infection consultation. For all analyses, the significance level was a priori set at $P<$ .05 . 
Buul, L.W. van, Veenhuizen, R.B., Achterberg, W.P., Schellevis, F.G., Essink, R.T.G.M., Greeff, S.C. de, Natsch, S., Steen, J.T. van der, Hertogh, C.M.P.M. Antibiotic prescribing in Dutch nursing homes: how appropriate is it? JAMDA: Journal of the American Medical Director Association: 2015, 16(3), 229-237

\section{Ethical Approval}

All study procedures were reviewed and approved by the Medical Ethics Review Committee of the VU University Medical Center (Amsterdam, The Netherlands) before study commencement.

\section{RESULTS}

\section{Demographics}

The 10 participating NHs had a mean of 163 beds per facility (range: 67-228) and a mean bed occupancy of $97 \%$ (range: $93 \%-100 \%$ ). On average, $51 \%$ of beds were for psychogeriatric patients (ie, mostly with dementia; range: $0 \%-78 \%$ ), 32\% for somatic patients (ie, with physical disability; range: $17 \%-72 \%$ ), and $17 \%$ for rehabilitation patients (range: $0 \%-35 \%$ ). In total, 707 consultations for 525 residents were registered by 62 physicians. Of these consultations, 406 (57\%) were for UTI, 247 (35\%) for RTI, and 54 (8\%) for SI.

Table 2 shows demographic characteristics of the residents. Residents had a mean age of 83.5, a median length of stay of 8 months, and were mostly women. Most residents were wheelchair-dependent, incontinent for urine, and diagnosed with dementia. There was substantial variation in case-mix among individual facilities, with significant differences for age, type of unit, proportion of wheelchair-dependent residents, urinary catheter use, proportion of residents with urinary incontinence, and proportion of residents with dementia.

\section{[TABLE 2. ]}

\section{APPROPRiATENESS OF TREATMENT Decisions}

Of the 707 registered consultations, sufficient information to evaluate the treatment decision was available for 598 cases (85\%; 90\% of UTI, 84\% of RTI, and $63 \%$ of SI). Antibiotics were prescribed in $88 \%$ of these cases. Overall, $76 \%$ of treatment decisions were judged appropriate, with significantly fewer appropriate treatment decisions for UTI (68\%) compared with RTI (87\%) and SI $(94 \%)(P<.001$; Table $3)$. Weighted for the number of cases per $\mathrm{NH}$, the same overall percentage of $76 \%$ appropriate treatment decisions was found (UTI, 70\%; RTI, 85\%; SI, 94\%).

Treatment decisions in which antibiotics were prescribed were less frequently judged appropriate (74\%) than decisions in which antibiotics were withheld $(90 \% ; P=$ .003). Further, facilities differed significantly in proportions of appropriate treatment decisions (range: 59\%-91\%; $P<.001$ ).

\section{[TABLE 3. ]}

We found lower proportions of appropriate prescribing decisions in residents of psychogeriatric units (72\%, versus $77 \%$ on somatic units and $83 \%$ on rehabilitation units; $P=.04$ ). As we found no differences in appropriate treatment decisions between $\mathrm{NH}$ units in a subgroup analysis per infection type, the overall difference is 
Buul, L.W. van, Veenhuizen, R.B., Achterberg, W.P., Schellevis, F.G., Essink, R.T.G.M., Greeff, S.C. de, Natsch, S., Steen, J.T. van der, Hertogh, C.M.P.M. Antibiotic prescribing in Dutch nursing homes: how appropriate is it? JAMDA: Journal of the American Medical Director Association: 2015, 16(3), 229-237

probably attributable to different patterns of infection types on different $\mathrm{NH}$ units (eg, relatively more UTI on psychogeriatric units). For RTI consultations, we found lower proportions of appropriate prescribing decisions in residents without COPD (83\%) compared with those with COPD (94\%; $P=.004)$. Other variables (ie, the physician, whether a resident was diagnosed with dementia, and whether a resident with UTI had a urinary catheter) were not significantly associated with appropriate prescribing.

Table 4 lists the most common clinical situations in which treatment decisions for UTI and RTI were considered inappropriate (SI was not included because of the low proportion of inappropriate treatment decisions). These all included situations in which antibiotics were prescribed. For UTI, the most common inappropriate prescribing was in cases that may involve asymptomatic bacteriuria. This occurred more frequently on psychogeriatric units (91\% of all inappropriate cases) than on somatic units (78\% of all inappropriate cases; $P=.03)$. For RTI, the most common inappropriate prescribing involved situations that suggest viral RTI. Inappropriate withholding of antibiotics occurred in only a few cases $(n=7)$. For UTI, these involved cases with a positive dipstick test (ie, the presence of nitrite and leukocyte esterase) in combination with specific urinary symptoms $(n=2)$, or nonspecific signs or symptoms in a patient who feels sick $(\mathrm{n}=3)$. For RTI, inappropriate withholding of antibiotics involved moderately ill $(n=1)$ and severely ill $(n=1)$ patients with COPD patients and acute cough.

\section{[TABLE 4. ]}

\section{CHART REVIEW}

Written informed consent for chart review was obtained for $56 \%$ of the invited residents (43\% to $73 \%$ per $\mathrm{NH}$ ). Charts of a total of 295 patients were reviewed (12 to 43 per $\mathrm{NH}$ ) over a mean period of 191 days (134 to 249 per NH). In total, 194 infection consultations ( 9 to 35 per $\mathrm{NH}$ ) were identified; in $59 \%$ of these cases (37\% to $78 \%$ per $\mathrm{NH}$ ), no registration form had been completed by physicians. Because of insufficient detailed information in patient charts, we were not able to assess appropriateness of the treatment decisions that had not been registered by physicians. We therefore compared other characteristics of these consultations with those that were registered, and found that nonregistered infections were less often treated with antibiotics (79\% versus $88 \%$ ), more often involved follow-up consultations (23\% versus $11 \%$ ), and were more often diagnosed and treated outside regular work hours by on-call physicians (18\% versus 11\%). Further, nonregistered infections were in patients with a longer median length of stay who less commonly resided on rehabilitation units. Other patient characteristics and the distribution of infection types (ie, 60\% UTI, 33\% RTI, and 7\% SI) were comparable between registered and nonregistered infections. There were no indications of overrepresentation of specific physicians among the nonregistered infections. 
Buul, L.W. van, Veenhuizen, R.B., Achterberg, W.P., Schellevis, F.G., Essink, R.T.G.M., Greeff, S.C. de, Natsch, S., Steen, J.T. van der, Hertogh, C.M.P.M. Antibiotic prescribing in Dutch nursing homes: how appropriate is it? JAMDA: Journal of the American Medical Director Association: 2015, 16(3), 229-237

\section{DisCUSSION}

We investigated the appropriateness of decisions to prescribe or withhold antibiotics in Dutch NHs and found that $76 \%$ of these decisions were appropriate. Treatment decisions were less often appropriate for UTI compared with decisions for RTI and SI. Decisions were more often appropriate when antibiotics were withheld compared with when antibiotics were prescribed, which indicates that overprescribing occurs more frequently than underprescribing. The most common clinical situations in which antibiotics were inappropriately prescribed were those indicative of asymptomatic bacteriuria and viral RTI.

The proportion of appropriate decisions to prescribe antibiotics (74\%) in our study is higher than reported in LTCF studies conducted in other countries (49\% to 63\%). ${ }^{6,7}$, 8, 9, 10 and 11 This may be explained by Dutch physicians being more conservative in antibiotic prescribing compared with physicians in other countries. ${ }^{16}$ and 20 This in turn may be related to country-specific characteristics regarding the societal context, physician training, and the organization of $\mathrm{NH}$ care (eg, the presence of on-site physicians, which enables them to get to know their patients well). ${ }^{20}$ and 22 Another possible explanation for the high proportion of appropriate treatment decisions is that the physicians' registration of infection consultations increased their awareness on appropriate antibiotic prescribing from the onset of data collection, resulting in higher proportions of appropriate antibiotic prescribing. Alternatively, other studies used chart review and may have underestimated appropriate prescribing if symptoms that justified antibiotic prescribing were not documented in the charts. Further, other algorithms may have been more stringent in evaluating appropriateness. However, some studies used the criteria of McGeer et $\mathrm{al}^{15}$ in assessing the appropriateness of antibiotic prescribing, 8 , 9 and 10 which have been developed for infection surveillance purposes and are therefore not highly sensitive, resulting in a relatively high risk of missing inappropriate cases. ${ }^{5}$ Other studies, ${ }^{6}$ and 7 similar to our study, used guidelinebased algorithms developed by an expert panel. The guideline used in these studies, however, dates back to 1971; we considered the minimum criteria developed by Loeb et $\mathrm{al}^{21}$ more up-to-date and therefore based our algorithm on these criteria, combined with criteria from national treatment guidelines.

Our finding that UTI was the most commonly occurring infection in LTCFs is in line with previous studies, as is our finding that antibiotics were most often inappropriately prescribed for this type of infection., 7, 9, 10, 23, 24 and 25 In addition, our study confirms that most of the inappropriate antibiotic prescribing for UTI is for asymptomatic bacteriuria, 7, 9, 10, 11 and 24 a situation for which antibiotic treatment is not beneficial. ${ }^{26}$ The prevalence of asymptomatic bacteriuria is high among LTCF residents, and consequently there is a high likelihood of obtaining positive results when performing a dipstick test. ${ }^{26,27}$ and 28 A dipstick test should therefore be performed only in case symptoms indicative of UTI are present, to rule out the diagnosis when negative. ${ }^{29}$ We found that clinical situations indicative of asymptomatic bacteriuria are more common on psychogeriatric units, where most residents have dementia. Diagnosis of infection is challenging in this population because of communication problems and the presentation of atypical symptoms. ${ }^{1}$ and ${ }^{21}$ For example, mental status change is a common reason to perform a dipstick test. ${ }^{12}$ 
Buul, L.W. van, Veenhuizen, R.B., Achterberg, W.P., Schellevis, F.G., Essink, R.T.G.M., Greeff, S.C. de, Natsch, S., Steen, J.T. van der, Hertogh, C.M.P.M. Antibiotic prescribing in Dutch nursing homes: how appropriate is it? JAMDA: Journal of the American Medical Director Association: 2015, 16(3), 229-237

The high prevalence of asymptomatic bacteriuria combined with the many other possible causes for mental status change are likely to result in substantial inappropriate antibiotic prescribing. This advocates for requiring the presence of additional signs and symptoms before performing a dipstick test in cognitively impaired residents with a change in mental status, especially as this patient group is more likely to acquire colonization with antibiotic-resistant pathogens compared with other residents. ${ }^{4}$

The finding that the proportion of appropriate prescribing in residents with COPD was higher than in those without COPD can be explained by national and international guidelines reflected in our algorithm, indicating a lower threshold for antibiotic prescribing in this group of patients. Further, in line with other findings, most of the RTI that we judged "inappropriate" were clinical situations that we considered indicative for viral RTI. ${ }^{9}$ and 24 The absence of one-sided abnormalities on lung auscultation often drove evaluation as inappropriate. This clinical sign is not considered in the criteria developed by Loeb et $\mathrm{al}^{21}$; however, it was given a central position in our algorithm based on a national guideline of the Dutch College of General Practitioners and consensus within the expert panel that contributed to the development of the algorithm. This is in agreement with 2 studies that reported abnormalities on lung auscultation to be predictors of pneumonia in patients in LTCFs and emergency departments. ${ }^{30}$ and 31 It may be argued that our algorithm should be liberalized due to the subjective nature of findings on lung auscultation, in which case more treatment decisions for RTI would have been classified "appropriate."

A strength of our study is that we assessed both decisions to prescribe and withhold antibiotics, whereas other studies on appropriateness of treatment decisions assessed only infections for which antibiotics were prescribed. 6, 7, 8, 9, 10 and 11 This enabled us to investigate the occurrence of both overprescribing and underprescribing. Another strength is that data collection was prospective and independent of availability of information in patient charts. The fact that we were not able to assess appropriateness of nonregistered infections due to incomplete information in patient charts, underlines the limitation of using patient charts.

Although registration of infection consultations by physicians thus resulted in more information per case compared with chart review, a limitation of this data collection method was that a substantial part of the infection consultations were not registered. This was at least partly due to physicians forgetting to complete a form in case the infection was diagnosed outside working hours, in case a form was recently completed for the same patient, and in case no antibiotic was prescribed. Another limitation is that we included only the decision to prescribe or withhold antibiotics in our evaluation of appropriateness of treatment decisions. Other elements of appropriate prescribing include, for example, selection of the right antibiotic drug, dose, and treatment duration. ${ }^{32}$

As studies evaluating appropriateness of antibiotic use in LTCFs so far have used different algorithms, the development of a universally applicable instrument would facilitate (international) comparison. Several existing guidelines and articles on 
Buul, L.W. van, Veenhuizen, R.B., Achterberg, W.P., Schellevis, F.G., Essink, R.T.G.M., Greeff, S.C. de, Natsch, S., Steen, J.T. van der, Hertogh, C.M.P.M. Antibiotic prescribing in Dutch nursing homes: how appropriate is it? JAMDA: Journal of the American Medical Director Association: 2015, 16(3), 229-237

appropriate indications for antibiotic treatment ${ }^{1,21,32,33,34 \text { and } 35}$ could be integrated into an instrument. For the development of such an instrument, it is important that applicability is ensured across LTCFs and nations, and in residents with dementia. ${ }^{12}$

Despite the relatively high proportion of appropriate antibiotic prescribing in the NHs in this study, the study findings indicate room for improvement in terms of reducing inappropriate treatment for asymptomatic bacteriuria and viral RTI. In 2 North American studies, interventions were reported that successfully reduced treatment for asymptomatic bacteriuria. ${ }^{28}$ and 36 In a qualitative study, we demonstrated that a variety of factors may be involved in antibiotic treatment decision-making, including use of diagnostic resources, physicians' perceived risks, influence of others, and influence of the environment (unpublished work by Van Buul LW, MSc, van der Steen JT PhD, Doncker SMMM, MSc, et al; 2014). Such factors may explain part of the observed differences in appropriateness of treatment decisions among facilities, and should therefore be considered in the development of interventions aimed at improving appropriate antibiotic prescribing in local settings.

\section{CONCLUSION}

Our findings suggest that more appropriate treatment decisions can lead to decreased antibiotic consumption in NHs in the Netherlands, as inappropriate treatment decisions were more often related to overuse than underuse of antibiotics. Appropriateness of treatment decisions can be improved by focusing on reduced antibiotic prescribing for asymptomatic bacteriuria, and to a lesser extent for viral RTI. Interventions directed at these conditions, thereby taking into account the many factors involved in antibiotic prescribing decision-making, are warranted to control antibiotic resistance in LTCFs.

\section{ACKNOWLEDGMENTS}

We acknowledge the following persons: Philip D. Sloane and Sheryl Zimmerman (University of North Carolina at Chapel Hill), for their contribution to the conception and design of the IMPACT study; Sarah M.M.M. Doncker, for her contribution to data collection; Giselka Gutschow, for her contribution to data processing; and Jochen W. Cals, Susanne E. Geerlings, Marlies E.J.L. Hulscher, Marianne A.B. van der Sande, Paul B.M. Went, Ellen E. Stobberingh, John E. Degener, Inge C. Gyssens, Theo J.M. Verheij, L.W. (Willem) Draijer, and Rogier M. Hopstaken for their contribution to the development of the algorithm for the evaluation of appropriateness of initiating or withholding antibiotics.

We thank the LTCFs for their participation in the study, in particular the physicians for their registration of infection consultations, and the residents and their family members for giving permission for chart review.

\section{APPENDIX}

Algorithms for the Evaluation of Appropriateness of Decisions to Prescribe or Withhold Antibiotics for Urinary Tract Infections, Respiratory Tract Infections, and 
Buul, L.W. van, Veenhuizen, R.B., Achterberg, W.P., Schellevis, F.G., Essink, R.T.G.M., Greeff, S.C. de, Natsch, S., Steen, J.T. van der, Hertogh, C.M.P.M. Antibiotic prescribing in Dutch nursing homes: how appropriate is it? JAMDA: Journal of the American Medical Director Association: 2015, 16(3), 229-237

Skin Infections (A, appropriate; AB, antibiotics; NA, not appropriate; PA, probably appropriate; PNA, probably not appropriate).

\section{REFERENCES}

1 L.E. Nicolle, D.W. Bentley, R. Garibaldi, et al. Antimicrobial use in long-term-care facilities. SHEA Long-Term-Care Committee Infect Control Hosp Epidemiol, 21 (2000), pp. 537-545

2 L.W. Van Buul, J.T. van der Steen, R.B. Veenhuizen, et al. Antibiotic use and resistance in long term care facilities J Am Med Dir Assoc, 13 (2012), pp. 568.e1-568.e13

$3 \mathrm{E}$. Nicolle, H.E. Sellers Resistant bacteria in nursing homes: A rational approach Hosp Pract (1995), 31 (1996), p. 15

4 A. Pop-Vicas, S.L. Mitchell, R. Kandel, et al. Multidrug-resistant gram-negative bacteria in a long-term care facility: Prevalence and risk factors J Am Geriatr Soc, 56 (2008), pp. $1276-1280$

5 D.A. Nace, P.J. Drinka, C.J. Crnich Clinical uncertainties in the approach to long-term care residents with possible urinary tract infection J Am Med Dir Assoc, 15 (2014), pp. 133-139

6 J.G. Zimmer, D.W. Bentley, W.M. Valenti, N.M. Watson Systemic antibiotic use in nursing homes. A quality assessment J Am Geriatr Soc, 34 (1986), pp. 703-710

$7 \mathrm{P}$. Montgomery, M. Semenchuk, L. Nicolle Antimicrobial use in nursing homes in Manitoba J Geriatr Drug Ther, 9 (1995), pp. 55-74

8 R.L. Stuart, J. Wilson, E. Bellaard-Smith, et al. Antibiotic use and misuse in residential aged care facilities Intern Med J, 42 (2012), pp. 1145-1149

9 C.J. Lim, S.C. McLellan, A.C. Cheng, et al. Surveillance of infection burden in residential aged care facilities Med J Aust, 196 (2012), pp. 327-331

10 M. Loeb, A.E. Simor, L. Landry, et al. Antibiotic use in Ontario facilities that provide chronic care J Gen Intern Med, 16 (2001), pp. 376-383

11 E.P. Peron, A.A. Hirsch, L.A. Jury, et al. Another setting for stewardship: High rate of unnecessary antimicrobial use in a Veterans Affairs long-term care facility J Am Geriatr Soc, 61 (2013), pp. 289-290

12 E. D'Agata, M.B. Loeb, S.L. Mitchell Challenges in assessing nursing home residents with advanced dementia for suspected urinary tract infections J Am Geriatr Soc, 61 (2013), pp. $62-66$

13 P. Rotjanapan, D. Dosa, K.S. Thomas Potentially inappropriate treatment of urinary tract infections in two Rhode Island nursing homes Arch Intern Med, 171 (2011), pp. 438-443

14 P. Vergidis, D.H. Hamer, S.N. Meydani, et al. Patterns of antimicrobial use for respiratory tract infections in older residents of long-term care facilities J Am Geriatr Soc, 59 (2011), pp. 1093-1098

15 A. McGeer, B. Campbell, T.G. Emori, et al. Definitions of infection for surveillance in longterm care facilities Am J Infect Control, 19 (1991), pp. 1-7

16 O. Cars, S. Mölstad, A. Melander Variation in antibiotic use in the European Union Lancet, 357 (2001), pp. 1851-1853

17 ESAC Nursing Home Collaborators. Report on point prevalence survey of antimicrobial prescription in European nursing homes, 2009. 2010. [report].

18 L.W. Van Buul, J.J. Sikkens, M.A. van Agtmael, et al. Participatory action research in antimicrobial stewardship: A novel approach to improving antimicrobial prescribing in hospitals and long-term care facilities J Antimicrob Chemother, 69 (2014), pp. 1734-1741

19 M.W. Ribbe, G. Ljunggren, K. Steel, et al. Nursing homes in 10 nations: A comparison between countries and settings Age Ageing, 26 (1997), pp. 3-12

20 J.T. Van der Steen, R.L. Kruse, M.E. Ooms, et al. Treatment of nursing home residents with dementia and lower respiratory tract infection in the United States and The Netherlands: An ocean apart J Am Geriatr Soc, 52 (2004), pp. 691-699

21 M. Loeb, D.W. Bentley, S. Bradley, et al. Development of minimum criteria for the initiation of antibiotics in residents of long-term-care facilities: Results of a consensus conference Infect Control Hosp Epidemiol, 22 (2001), pp. 120-124

22 M.R. Helton, J.T. van der Steen, T.P. Daaleman, et al. A cross-cultural study of physician treatment decisions for demented nursing home patients who develop pneumonia

Ann Fam Med, 4 (2006), pp. 221-227 
Buul, L.W. van, Veenhuizen, R.B., Achterberg, W.P., Schellevis, F.G., Essink, R.T.G.M., Greeff, S.C. de, Natsch, S., Steen, J.T. van der, Hertogh, C.M.P.M. Antibiotic prescribing in Dutch nursing homes: how appropriate is it? JAMDA: Journal of the American Medical Director Association: 2015, 16(3), 229-237

23 S.R. Jones, D.F. Parker, E.S. Liebow, et al. Appropriateness of antibiotic therapy in longterm care facilities Am J Med, 83 (1987), pp. 499-502

24 J.W. Warren, F.B. Palumbo, L. Fitterman, S.M. Speedie Incidence and characteristics of antibiotic use in aged nursing home patients J Am Geriatr Soc, 39 (1991), pp. 963-972

25 P.R. Katz, T.R. Beam Jr., F. Brand, K. Boyce Antibiotic use in the nursing home

Arch Intern Med, 150 (1990), pp. 1485-1488

26 L.E. Nicolle Urinary tract infection in long-term-care facility residents Clin Infect Dis, 31 (2000), pp. 757-761

27 S. Walker, A. McGeer, A.E. Simor, et al. Why are antibiotics prescribed for asymptomatic bacteriuria in institutionalized elderly people? A qualitative study of physicians' and nurses' perceptions CMAJ, 163 (2000), pp. 273-277

28 T.F. Zabarsky, A.K. Sethi, C.J. Donskey Sustained reduction in inappropriate treatment of asymptomatic bacteriuria in a long-term care facility through an educational intervention

Am J Infect Control, 36 (2008), pp. 476-480

29 M. Juthani-Mehta, M. Tinetti, E. Perrelli, et al. Role of dipstick testing in the evaluation of urinary tract infection in nursing home residents Infect Control Hosp Epidemiol, 28 (2007), pp. 889-891

30 D.R. Mehr, E.F. Binder, R.L. Kruse, et al. Clinical findings associated with radiographic pneumonia in nursing home residents J Fam Pract, 50 (2001), pp. 931-937

31 P.S. Heckerling, T.G. Tape, R.S. Wigton, et al. Clinical prediction rule for pulmonary infiltrates Ann Intern Med, 113 (1990), pp. 664-670

32 I.C. Gyssens, P.J. van den Broek, B.J. Kullberg, et al. Optimizing antimicrobial therapy. A method for antimicrobial drug use evaluation J Antimicrob Chemother, 30 (1992), pp. 724727

33 C.J. Lim, D.C.M. Kong, R.L. Stuart Reducing inappropriate antibiotic prescribing in the residential care setting: Current perspectives Clin Interv Aging, 9 (2014), pp. 165-177

34 S. Zimmerman, P.D. Sloane, R. Bertrand, et al. Successfully reducing antibiotic prescribing in nursing homes J Am Geriatr Soc, 62 (2014), pp. 907-912

35 K.P. High, S.F. Bradley, S. Gravenstein, et al. Clinical practice guideline for the evaluation of fever and infection in older adult residents of long-term care facilities: 2008 update by the Infectious Diseases Society of America J Am Geriatr Soc, 57 (2009), pp. 375-394

36 M. Loeb, K. Brazil, L. Lohfeld, et al. Effect of a multifaceted intervention on number of antimicrobial prescriptions for suspected urinary tract infections in residents of nursing homes: Cluster randomised controlled trial BMJ, 331 (2005), p. 669

\section{TABLES}

Table 1

Recruitment of Study Facilities

\begin{tabular}{|c|c|c|}
\hline Approached & Agreed & Reasons for Refusal \\
\hline 9 individual $\mathrm{NHs}$ & $6 \mathrm{NHs}$ (2 affiliated with the same health care organization) & Organizational issues (2), unknown (1) \\
\hline 3 health care organizations & 1 health care organization ( 3 of 4 affiliated NHs signed up for participation) & Unknown (2) \\
\hline $\begin{array}{l}1 \text { university-affiliated network of } \\
7 \text { health care organizations }\end{array}$ & 1 affiliated NH signed up for participation & - \\
\hline
\end{tabular}


Buul, L.W. van, Veenhuizen, R.B., Achterberg, W.P., Schellevis, F.G., Essink, R.T.G.M., Greeff, S.C. de, Natsch, S., Steen, J.T. van der, Hertogh, C.M.P.M. Antibiotic prescribing in Dutch nursing homes: how appropriate is it? JAMDA: Journal of the American Medical Directore Association: 2015, 16(3), 229-237

Table 2

Resident Characteristics of Registered Infection Consultations

\begin{tabular}{lc}
\hline Characteristic & Infection Consultations, $\mathrm{n}=707$ \\
\hline Sociodemographic & \\
Female, n/N (\%) & $511 / 707(72.3)$ \\
Age; n, mean (range) & $703,83.5(43.0-101.0)$ \\
Length of stay, mo, n, median (range) & $649,8.0(0.0-191.0)$ \\
Type of unit, n/N (\%) & \\
$\quad$ Somatic & $260 / 705(36.9)$ \\
Psychogeriatric & $318 / 705(45.1)$ \\
$\quad$ Rehabilitation & $127 / 705(18.0)$ \\
Functioning, n/N (\%) & \\
Wheelchair-dependent & $374 / 658(56.8)$ \\
Urinary catheter & $106 / 671(15.8)$ \\
Urinary incontinence* & $447 / 595(75.1)$ \\
Comorbidities, n/N (\%) & \\
Diabetes mellitus & $133 / 682(19.5)$ \\
Chronic obstructive pulmonary disease & $108 / 676(16.0)$ \\
Dementia & $340 / 657(51.8)$ \\
\hline
\end{tabular}

'The physicians sometimes did not know whether a resident was incontinent for urine or not, which explains the relatively high number of missing cases (ie, 112) on this variable.

Table 3

Proportion of Appropriate Treatment Decisions for Residents With UTI, RTI, and SI

Appropriate Treatment Decisions, $\mathrm{n} / \mathrm{N}$, \% (Range Across Facilities)

\begin{tabular}{cc}
\hline Overall & $453 / 598,75.8(58.6-91.3)$ \\
UTI & $241 / 356,67.7(53.5-89.3)$ \\
RTI & $180 / 208,86.5(60.0-96.2)$ \\
SI & $32 / 34,94.1(66.7-100.0)$ \\
\hline
\end{tabular}


Buul, L.W. van, Veenhuizen, R.B., Achterberg, W.P., Schellevis, F.G., Essink, R.T.G.M., Greeff, S.C. de, Natsch, S., Steen, J.T. van der, Hertogh, C.M.P.M. Antibiotic prescribing in Dutch nursing homes: how appropriate is it? JAMDA: Journal of the American Medical Directors Association: 2015, 16(3), 229-237

Table 4

Clinical Situations that Represent $>10 \%$ of the Inappropriate Treatment Decisions

\begin{tabular}{|c|c|}
\hline$\%$ of the Inappropriate Treatment Decisions & Description of Clinical Situation \\
\hline \multicolumn{2}{|l|}{ UTls ( $\mathrm{n}=90$ inappropriate treatment decisions) } \\
\hline $50.0 \%$ & $\begin{array}{l}\text { Antibiotic treatment for a patient without a urinary catheter, who does not feel sick, and has no delirium or } \\
\text { specific symptoms, but has aspecific symptoms (eg, suprapubic pain, confusion) in combination with a } \\
\text { positive nitrite and leukocyte esterase test. }\end{array}$ \\
\hline $18.9 \%$ & $\begin{array}{l}\text { Antibiotic treatment for a patient without a urinary catheter, who has no specific symptoms, and a negative } \\
\text { nitrite test, but has aspecific symptoms (eg, suprapubic pain, confusion) in combination with a positive } \\
\text { leukocyte esterase test. }\end{array}$ \\
\hline $11.1 \%$ & $\begin{array}{l}\text { Antibiotic treatment for a patient without a urinary catheter, who does not feel sick, has no delirium, and a } \\
\text { negative nitrite test, but has specific symptoms (eg, dysuria, frequency) in combination with a positive } \\
\text { leukocyte esterase test. }\end{array}$ \\
\hline \multicolumn{2}{|l|}{ RTIs ( $n=20$ inappropriate treatment decisions) } \\
\hline $45.0 \%$ & $\begin{array}{l}\text { Antibiotic treatment for a patient with acute cough who is moderately ill or has fever }\left(\geq 38^{\circ} \mathrm{C}\right) \text {, but has no } \\
\text { COPD or one-sided abnormalities on lung auscultation. }\end{array}$ \\
\hline $15.0 \%$ & $\begin{array}{l}\text { Antibiotic treatment for a moderately ill patient without cough, but with fever }\left(\geq 38^{\circ} \mathrm{C}\right) \text {, possibly combined } \\
\text { with delirium, but without tachypnea, COPD, or one-sided abnormalities on lung auscultation. }\end{array}$ \\
\hline
\end{tabular}

